\title{
High-performance nanoporous silicon-based photodetectors
}

\begin{abstract}
A series of porous silicon (PSi) samples was prepared using photoelectrochemical etching (PECE) method with optimum current density of $45 \mathrm{~mA} / \mathrm{cm} 2$. The as-prepared PSi samples were characterized to determine the influence of the etching time $(15,25$ and $30 \mathrm{~min})$ on their morphology and electrical properties. The percentage of porosity was estimated via gravimetric analysis. The band gap of the fabricated PSi was $\approx 2.22 \mathrm{eV}$. Upon their use to fabricate metal-semiconductor-metal (MSM) ultraviolet photodetectors (UVPD), the fabricated PSi revealed excellent stability and reliability under repetitive shots at $530 \mathrm{~nm}$. Furthermore, very fast rise time $(\approx 0.28 \mathrm{~s})$ was obtained at a bias of $1 \mathrm{~V}$ under visible light $(530 \mathrm{~nm})$ illumination.
\end{abstract}

Keyword: Porous silicon; Etching time; Electrical properties; Photodetector 